\title{
DISCUSSION
}

\section{Dilatancy for cohesionless soils}

\author{
X. S. LI and Y. F. DAFALIAS (2000). Géotechnique 50, No. 4, 449-460
}

\section{A. Vatsala. Indian Institute of Science, Bangalore}

The authors have presented a very effective and, at the same time, very simple relation to describe the dilatancy of cohesionless soils, which is applicable for the whole range of densities and loading conditions. The final expression proposed shows the dilatancy to be a function of the stress ratio, $\eta$, and the state parameter $\psi$, in addition to some material constants such as $M$, $d_{0}$ and $m$. Although they mention the possible influence of some internal state variables such as the evolving tensor of anisotropy, this was not included in the final form. This discussion is intended to bring out the significant influence of certain internal variables, the negligence of which may lead to unsatisfactory predictions, especially during reverse and cyclic loading.

We feel the contractancy of loose samples or the dilatancy of dense samples beyond the phase transformation is in accordance with critical-state concepts and hence can be related to the state parameter $\psi$, whereas the initial contractancy of a dense sample or the contractancy during shear unloading is not in accordance with critical-state concepts and hence may not be related only to $\psi$. Two soil states with the same state parameter and at the same stress level can show different dilatancy depending on their previous stress history. For example, Fig. 16 shows two samples, $\mathrm{A}$ and $\mathrm{B}$, at the same density and the same stress state with $\eta \approx M$. Sample A, being relatively dense, was moving towards the critical state beyond the phase transformation stage during undrained shearing. Sample B has had a cyclic shear loading history leading to near liquefaction and has then been loaded up to the current stress. The two samples are unloaded at this stage. Sample A would show a gentle decrease in $p^{\prime}$ while sample B would show a more drastic decrease with a reverse path almost following $\eta=M$. A cyclically pre-sheared sample would similarly show greater contractancy during loading. These differences in behaviour are obviously due to differences in the internal variables of the two states.

Recent studies with discrete element modelling can give a complete record of the internal variables. Although there are several results for drained shearing, results for undrained shearing DEM simulations are limited. Important findings from such DEM simulations (Ng Tang-Tat, 1989; Nagaraja Rao, 1998; Sitharam et al., 1999) are that there is a continuous decrease in the number of contacts or coordination number, as well as in the average contact force during undrained shearing, within the phase transformation stage, whereas, during merely isotropic unloading (probably during any constant $\eta$ unloading), there is not much decrease in the number of contacts but a greater decrease in the average contact force magnitude. The number of contacts and the average contact force together contribute to the mean normal stress, $p^{\prime}$, of the assembly.

The rate of dilatancy appears to be related to the number of contacts available in relation to the current stress, $p^{\prime}$. If the number of contacts is just sufficient to carry the current stress, $p^{\prime}$, shearing at constant volume-that is, $\mathrm{d} \eta$-will result in a greater loss of contacts, or a reduction in $p^{\prime}$ (or positive pore pressure). If the available number of contacts is more than required, the rate of loss of contacts and hence the effective change will be smaller (the former happens in loose or presheared states, and the latter in denser states and for states well within failure). Thus there is a need to study the pattern of variation of number of contacts even at constant volume, and to define dilatancy in relation to this coordination number. Not only the dilatancy, but also other features such as the virtual peak stress ratio and hence the plastic modulus, $K_{\mathrm{p}}$, also appear

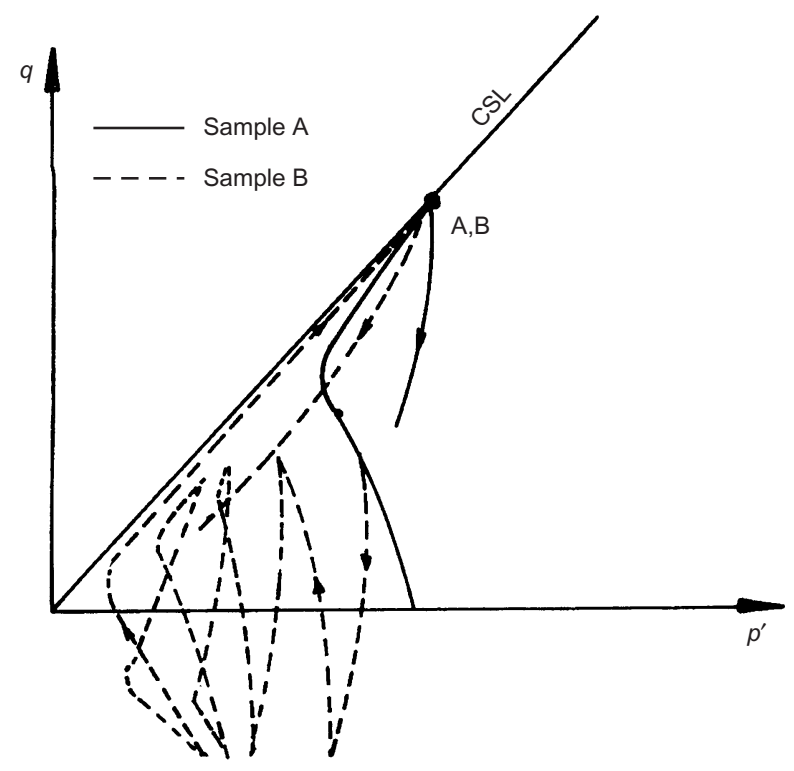

(a)

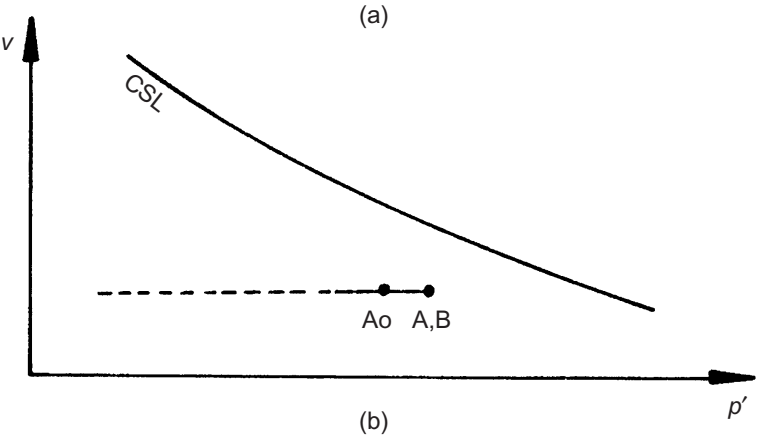

Fig. 16. Schematic diagram showing samples with the same state parameter exhibiting different responses during unloading

to be related to the current coordination number. It may even be possible in future to define the yield curves and their hardening also in terms of this internal state parameter, when the entire behaviour may follow a simple associated flow rule. Basically, within the phase transformation, the response is one of small strain. Here the system attempts to achieve the required anisotropy in contacts and contact forces within the available number of contacts, without much rearrangement of particles. The easiest way in which this can be achieved is by loss of contacts and contact forces in the lateral direction. This can proceed up to a limiting stress ratio without there being excessive shear forces. This could be the phase transformation stage. Beyond this, major particle rearrangements brought about by large strains will be required to acquire configurations suitable to carry the applied deviator loading. The loss of contacts within the phase transformation stress ratios continues to occur cumulatively over cycles of stress reversals as long as the stress ratio remains within the limiting value, thus leading to liquefaction. That is, the contractancy in this stage is dictated not by the critical void ratio but by a pseudo-critical state corresponding to the available number of contacts at that instant. The loss of 
contacts may not involve shearing at contacts with dissipation of plastic energy but may involve only decrease of normal stress up to zero, for many of the contacts, unlike the post-phasetransformation stage. This is reflected as a higher contractancy and a higher plastic modulus.

\section{Authors' reply}

The authors greatly appreciate this insightful discussion of their paper, which indeed brings up a number of interesting issues. To focus on the main theme, the paper in question addresses only the sand response under monotonic drained and undrained loading. Had the cyclic loading been considered, the observations by the discusser would have been addressed, at least qualitatively.

It is clear that these observations pertain to the formation and/or change of fabric during cyclic loading. To a certain extent such a fabric change was considered macroscopically in earlier papers by Manzari \& Dafalias (1997), mentioned by the discusser, and Li et al. (1999), with the introduction of kinematic hardening and reversal stress point concepts for cyclic loading, reflecting the locked-in stress and effect of the distribution of contact normals in reverse loading events, as well as the change from dilatant to contractant response when reverse loading takes place for stress ratios greater than the phase transformation stress ratio. In fact this point of reverse dilatancy is a much-neglected aspect of many constitutive models, and cannot be derived from Rowe's fundamental dilatancy theory, which was based on observations under monotonic loading only. That is why it was necessary to introduce the concept of a dilatancy surface in stress space, similar to that of a failure surface (in Li et al. (1999) these two surfaces coincided), such that the dilatancy at each stage depends on the distance of the current stress ratio from an image point on the dilatancy surface, thus generalising Rowe's concept to any event of loading or reverse loading.

Despite this formulation, the discusser's observations shown in Fig. 16 are reminders that the state parameter $\psi$ alone, being a scalar-valued quantity, cannot possibly account for anisotropic fabric changes during cyclic loading, even in the presence of kinematic hardening, which is an anisotropic variable good in accounting only up to a certain point for fabric changes. For the same reason the number of available contact points (coordination number), a scalar-valued entity also, must be supplemented by the corresponding distribution of orientations of contact normals, in order to be a meaningful internal variable for proper fabric considerations. It is possible that, for the discrete element method of analysis, such an orientational distribution is not needed, for the mere reason that the method keeps track of the response of each particle separately. But for a continuum mechanics analysis such a distribution or its corresponding macroscopic counterpart is a necessity.

In this respect work has been under way. In particular the schematic response shown in Fig. 16 is both dramatic and important, since it can lead to liquefaction, while showing that, at the point of unloading, the two samples A and B, while having the same stress and state parameter values, do respond quite differently. This motivated a recent work by Dafalias \& Manzari (1999) in which a variable fabric dilatancy tensor is introduced in the formulation, which macroscopically accounts by its norm for the change in the number of contact points (although not specified as such), and by its direction for the change in the orientational distribution of contact normals, particularly in the dilatant phase when the stress ratio is above the phase transformation value, in accordance with microscopic observations by Nemat-Nasser \& Tobita (1982). The approach is simple and phenomenological, and the role of this fabric dilatancy tensor is to enhance the contractive tendencies upon unloading following a dilatant phase. The greater is the dilatant phase preceding unloading (e.g. sample B compared with sample A in Fig. 16), the greater is the contractancy in unloading. This concept can straightforwardly be introduced in the model under discussion when unloading is considered, as well as in other similar models, and supplements the role of the state parameter $\psi$.

It should also be noted that the slope of the undrained $p^{\prime}-q$ stress path is related not only to the dilatancy but also to the plastic modulus. In fact, the slope in terms of $\mathrm{d} p^{\prime} / \mathrm{d} \eta$ is proportional to the dilatancy but inversely proportional to the plastic modulus. A reduction of plastic modulus, which is most likely associated with a reducing coordination number during previous shearing cycles, may also lead to a faster decrease in mean normal stress, $p^{\prime}$, as highlighted by the discusser in Fig. 16 for the differences in stress paths for the samples A and B. This loading history dependence of plastic modulus has been well reflected by the authors in a generalised sand model following the state dependent dilatancy concept. In that model, the plastic modulus is a function of the accumulated plastic loading index.

\section{REFERENCES}

Dafalias, Y. F. \& Manzari, M. T. (1999). Modeling of fabric effect on the cyclic loading response of granular soils. CDROM Proc., 13th ASCE Engng. Mech. Div. Conf., Baltimore, 00-00?.

Li, X. S., Dafalias, Y. F. \& Wang, Z. L. (1999). State dependent dilatancy in critical state constitutive modeling of sand. Can. Geotech. J. 36, No. 4, 599-611.

Manzari, M. T. \& Dafalias, Y. F. (1997). A critical state two-surface plasticity model for sands. Géotechnique 47, No. 2, 255-272.

Nagaraja Rao (1998). Discrete element simulation of liquefaction behaviour of sands. MSc dissertation, Indian Institute of Science, Bangalore, India.

Nemat-Nasser and Tobita (1982). Influence of fabric on liquefaction and densification potential of cohesionless sand. Mech. Mater. 1, 43-62.

$\mathrm{Ng}$ Tang-Tat (1989). Numerical simulation of granular soil under monotonic and cyclic loading: a particulate mechanics approach. $\mathrm{PhD}$ dissertation, Rensselaer Polytechnic Institute, New York, USA.

Sitharam, T. G., Vatsala, A. \& Nagaraja Rao, P. V. (1999). Numerical simulation of cyclic behaviour of sands using discrete element modelling. Geotech. Engng Bull. 8, 11-39. 